\title{
STUDY OF COSMIC DUST PARTICLES ON BOARD LDEF AND MIR SPACE STATION
}

\author{
J.C. MANDEVILLE \\ ONERA/CERT Space Technology Department \\ P.O.BOX 4025 \\ 31055 Toulouse Cedex \\ France
}

\begin{abstract}
Interplanetary and near-earth space contains solid objects whose size distribution continuously covers the interval from submicron sized particles to $\mathrm{km}$ sized asteroids or comets.

Two French experiments partly devoted to the detection of cosmic dust have been flown recently in space. One on the NASA Long Duration Exposure Facility (LDEF), and one on the Soviet MIR Space Station. A variety of sensors and collecting devices will make possible the study of cosmic particles after recovery of exposed material. Flux mass distribution is expected to be derived from craters counts, with a good accuracy. Remnants of particles, suitable for chemical identification are expected to be found within stacked foil detectors. Discrimination between extraterrestrial particles and man-made orbital debris will be possible.
\end{abstract}

\section{Introduction}

Our present knowledge of the occurence and of the physical properties of micrometeoroids is based primarly on earth bound observation of meteors, comets, zodiacal light, data from infrared satellites (IRAS) as well as on board measured flux by instrumented spacecrafts (Pegasus, Explorers, Vega, Giotto, Space Shuttle ), study of lunar samples and dust collection in upper atmosphere (Leinert and Grün, 1990). Part of meteoroids originate from comets (mainly dust ejected at perihelion), part originate from collisions within asteroid belt. The relative contribution of these two sources is still a matter of debate, a majority of particles are likely coming from comets but recent data from Infrared Astronomy Satellite (IRAS) indicate than asteroids could be a source larger than expected. In addition to natural particles, a significant and growing number of particles has been added by human activity, in near earth space.

Extensive modeling of the distribution of dust particles has been made, and the NASA 1969 Meteoroid model (Cour-Palais, 1969) is still widely accepted. Recent modeling for artificial debris has been proposed by D. Kessler (Kessler et al.,1989). Data from recent space experiments could possibly lead to a reassessment of earlier models (Alexander et al.,1963).

Originally launched in April 1984 for a nine months mission the NASA Long Duration Exposure Facility (LDEF) has been retrieved, in January 1990, after 2105 days in terrestrial orbit, between 450 and $330 \mathrm{~km}$ altitude.

The MIR Soviet Space Station has been in a $350 \mathrm{~km}$ circular orbit since February 1986. It is composed of a main module (15 meter long, 4 meter in diameter), associated with other specialised modules. The French experiment, named "Echantillons" was deployed outside the station, during the Aragatz Mission in december 1988; it was retrieved 13 months later.

A.C. Levasseur-Regourd and H. Hasegawa (eds.), Origin and Evolution of Interplanetary Dust, 11-14. (1991 Kluwer Academic Publishers, Printed in Japan. 


\section{Experimental Approach}

Part of the LDEF tray allocated to french experiments, known as FRECOPA payload, has been devoted to the study of dust particles. The tray was located on the face of LDEF directly opposed to the velocity vector (west facing direction, location B3, according to LDEF description).

Two passive experiments have been flown : one composed of a set of glass and metallic samples and one composed of multilayer thin foils detectors. Collection area was about $2000 \mathrm{~cm}^{2}$. In addition of these dedicated experiments a broad variety of materials has been exposed to the bombardment of microparticles and are expected to provide additional data. Thick target experiment comprises selected metallic (Al,Au, $\mathrm{Cu}, \mathrm{W}$, Stainless Steel, thickness: $250 \mu \mathrm{m}$ ) and glass surfaces $1.5 \mathrm{~mm}$ thick.

Crater size distribution from these thick target experiments will enable, with the aid of laboratory calibration by solid particle accelerators, the evaluation of the incident microparticle flux in the near earth environment. A more critical issue is the determination of the chemical composition of the impacting particles. In general they are physically destroyed and mixed with target material in the process of crater formation, however chemical identification of remnants has proven possible (Warren et al.,1989).

The aim of the multiple foil penetration and collection experiment is primarly to investigate the feasability of multilayer thin film detectors acting as energy sorters in order to collect micrometeoroids, if not in their original shape, at least as "break-up" fragments suitable for chemical analysis.

Foil thickness ranges from $0.75 \mu \mathrm{m}$ to $5 \mu \mathrm{m}$ of aluminium; such foils are expected to slow down particles with diameters between 1 and $10 \mu \mathrm{m}$ diameter, without complete destruction.

The experiment on MIR carried basically the same passive sensors, with an addition, an active dust detector. This detector was based on the monitoring of the discharge upon impact of a thin foil capacitor. Due to the stabilization mode of the station, sensors were facing the velocity vector direction during aproximately half of the orbit. Detailed description of hardware has been given elsewhere (Mandeville, 1984, Mandeville and McDonnell, 1984).

\section{Preliminary Results.}

Both experiments have been recovered in good conditions after exposure to space. As expected, the number of impact craters vary significantly with the location on spacecraft surface (with respect to velocity vector). During its mission LDEF was stabilized with the long axis continually pointed toward the center of the earth, and surfaces perpendicular to this axix pointed at fixed angles with respect to the direction of orbital motion. Preliminary comparison with data from similar experiments located on different sides of LDEF has been made. Materials not specifically dedicated to dust detection have provided usefull data, mostly because the large area time exposure. The spacecraft has been the first one purposely designed to make possible an extensive study of space environment, in peculiar micrometeoroids, after a recovery of the experiments. The detection range goes from submicron to $\mathrm{mm}$ sized particles.

Two large large impact features on the Frecopa tray (LDEF) have been found : one full penetration and one marginal penetration of a $1 \mathrm{~mm}$ aluminium shield. About 100 craters larger than 30 microns have been found on a area of $1 \mathrm{~m}^{2}$. Most of the large craters are circular in outline, however some small craters do indicate oblique incidence. SEM investigation for small craters is still in progress but preliminary results do indicate apparently a deficiency in micron sized craters. First results from the largest craters on the S0001 Space Debris Impact Experiment (D.Humes) are shown by courtesy of M-D SIG Team (See et al.,1990), in comparison with our data. The impact site survey yields a crater size distribution, which should be converted to a particle mass distribution by using relevant relationships between crater sizes and particles mass and velocity. The discussion is out of the scope of this paper, however assuming an average impact velocity of $15 \mathrm{~km} / \mathrm{s}$, the value of the crater diameter to the particle diameter ratio, could be chosen as $\mathrm{D} / \mathrm{d}=5$, for aluminium targets.

The figure 1 shows the preliminary size distribution of craters found on some detectors for the LDEF and for the MIR experiments. Detailed SEM analysis has been made on a few aluminium targets. A large density of submicron craters has been found on detectors exposed on the MIR station. The data indicate the presence of particles with a diameter of a few tenths of microns (mass of $10^{-15} \mathrm{gm}$ ) in earth bound orbits. Data from IDE experiment on LDEF are consistent with these results (Singer et al.,1990), as well as measurements reported by Singer (Singer and Stanley,1980). 
The size distribution shows a good agreement with comparable near-earth data obtained by McDonnell (McDonnell et al. 1991); flux on the west face of LDEF is about 20 times lower than on the east face, for large particles. Most of the particles impacting this face should be interplanetary dust particles, not orbital debris. This fact will be substantiated further by the chemical identification of projectile remnants inside craters.

Comparison MIR/LDEF/NASA Model

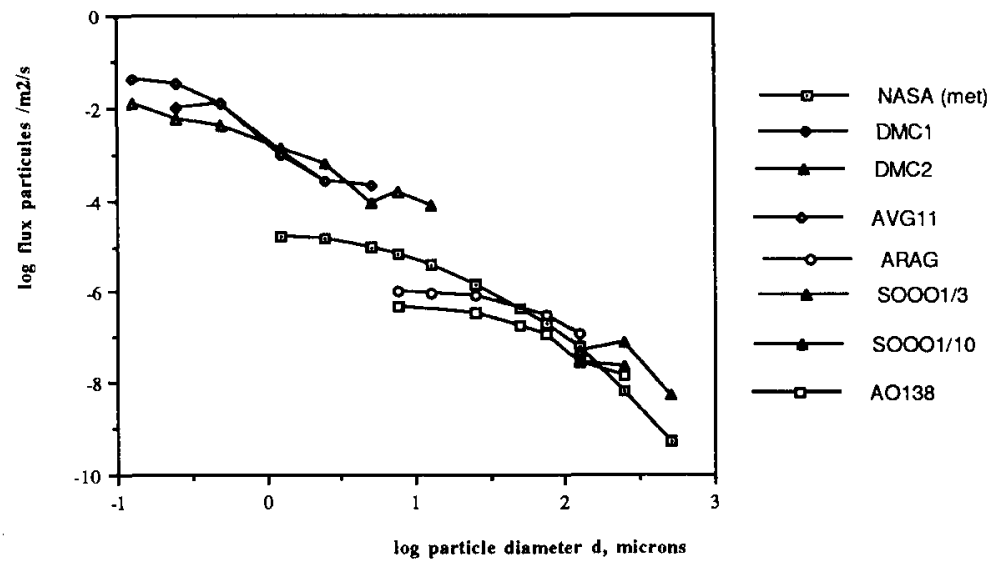

Figure 1. Flux (particles $/ \mathrm{m}^{2} / \mathrm{s}$ ) of dust particles versus diameter (microns). Comparison between space experiments (near earth) and NASA model is shown. Symbols are as follows :

NASA(met) is the NASA 1969 Meteoroid model.

DMC1, DMC2 and AVG11 are data from dust detectors on MIR (for small particles).

ARAG are data from MIR experiment (for large particles).

AO138 are data from LDEF FRECOPA experiment (for large particles)

S0001/3 and S0001/10 are data from NASA Langley (D. Humes) LDEF dust experiment (Large particles, west and east faces, respectively).
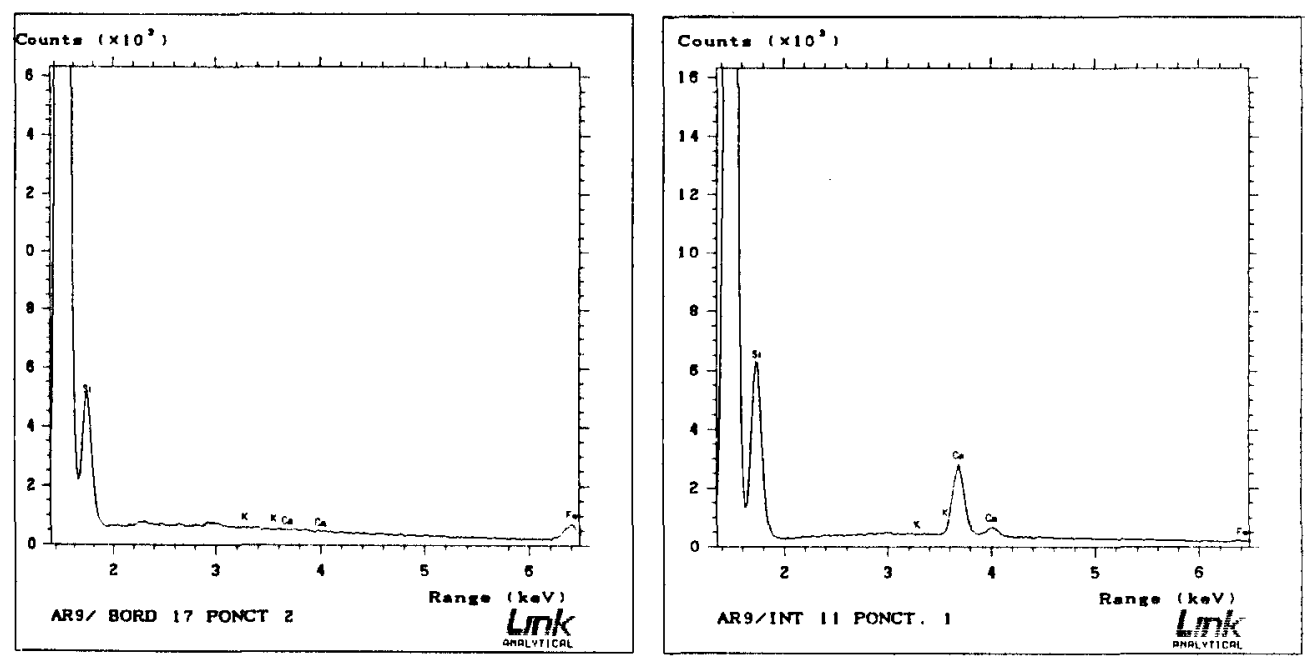

Figure 2. Energy dispersive X-Ray spectra from points located inside impact craters 
The slope of the size distribution of particles is consistent with data obtained elsewhere on LDEF, the change of slope at large sizes could be an artifact due to the limited time-area of sampling, or more likely real as shown by McDonnell .

As the inclination of the orbit of the MIR station is $58^{\circ}$, a comparison with LDEF data (inclination $28^{\circ}$ ) is expected to provide some information on the orbital distribution of artificial debris. Preliminary results indictate a somewhat higher flux of micron-sized particles on MIR orbit.

The first $\mathrm{X}$ ray analysis of some small craters has shown evidence of elements $\mathrm{Ca}, \mathrm{K}, \mathrm{Ti}, \mathrm{Fe}$ and $\mathrm{S}$. The figure 2 shows the occurence of calcium and of iron inside two small craters found on MIR samples. Detailed analysis will doubtless give insight on the origin of impacting objects and some information on the relative contribution of cosmic dust particles and man-made debris.

\section{Conclusion.}

As shown by the preliminary investigation of experiments and materials retrieved on LDEF and on MIR station, better knowledge of the micrometeoroid environment in near-earth orbits will be obviously obtained from dedicated in-flight, retrievable experiments. Large time area exposure of detectors will make possible reliable statistics on size distribution of particles. Discrimination between man-made orbital debris and cosmic particles is a difficult but necessary task in order to assess the extent of the pollution of near space.

Collection in space of undisturbed particles will remain generally difficult, perhaps impossible for the highest meteoritic velocities. However the lower velocity window for multiple layer foil deceleration would prove sufficient to expect the retention of material suitable for identification. Investigation of the near-earth region of space is a necessity as well as for scientific or technical purposes.

\section{REFERENCES.}

Alexander, W.M. et al. (1963) 'Review of direct measurements of interplanetary dust from satellites and probes', in W. Priester (ed.), Proc. Third Intern. Space Sci.Symp., J.Wiley Publishers, p.891.

Cour-Palais, B.G. (1969) 'Meteoroid environment model', NASA SP 8013.

Kessler, D.J., Reynolds, R.C. and Anz-Meador, P.D. (1989) 'Orbital debris environment', NASA TM 100471

Leinert, Ch. and Grün, E. (1990) 'Interplanetary Dust', in Physics and Chemistry in Space, Springer Publishers, (in press).

Mandeville, J-C. and McDonnell, J.A.M. (1980) 'Micrometeoroid multiple foil penetration and particle recovery experiments on LDEF', in IAU Symposium 90 Proceedings, D. Reidel Publishers, Dordrecht.

Mandeville, J-C. (1984) 'AO138-1 and AO138-2 Experiments', in L.G.Clark et al. (eds.), LDEF Mission 1 Experiments, NASA SP-473.

Mandeville, J-C. (1990) 'Aragatz Mission Dust Collection Experiment', Adv.Space Res. 10, pp.397401.

McDonnell, J.A.M. et.al. (1991) 'Particulate detection in the near earth space environment aboard the long duration exposure facility LDEF: Cosmic or Terrestrial', Proc. IAU Coll. on 'Origin and Evolution of Interplanetary Dust', Kyoto, Japan.

See T., et al. (1990). 'Meteoroid and debris impact features documented on the Long Duration Exposure Facility', M-D SIG, Prelim.Report.,NASA.

Singer, S.F. et al. (1990) 'First spatio-temporal results from the LDEF Interplanetary dust experiment (IDE)', Preprint XXVIII COSPAR 1990.

Singer, S.F. and Stanley, J.E. (1980) 'Submicron particles in meteor streams', in IAU Symposium 90 proceedings, D.Reidel Publishers, Dordrecht.

Warren, J.L. et al. (1989) 'The detection and observation of meteoroid and space debris impact features on the Solar-Max satellite', in Proceedings XIXth Lun.Plan.Sci.Conf., pp. 641-657.

Acknowledgements.

Support from CNES (F) and Glavcosmos (USSR) for experiments on the MIR Space Station and from NASA (USA) for experiments on LDEF is greatly acknwoledged. 